\title{
NOUVELLE
}

\section{Espoirs thérapeutiques dans la dégénérescence maculaire liée à l'âge}

Francine Behar-Cohen, Florian Sennlaub, Marianne Berdugo
F. Behar-Cohen : Inserm U598, Physiopathologie des maladies oculaires, Innovations thérapeutiques, Centre de Recherches Biomédicales des Cordeliers,

15, rue de l'École de Médecine, 75270 Paris Cedex 06, France. Service d'ophtalmologie, Hôtel-Dieu,

1, place du Parvis de Notre Dame, 75004 Paris, France. behar@idf.inserm.fr

F. Sennlaub, M. Berdugo: Inserm U598, Physiopathologie des maladies oculaires, Innovations thérapeutiques, Centre de Recherches Biomédicales des Cordeliers, 15, rue de l'École de Médecine, 75270 Paris Cedex 06, France.
> La dégénérescence maculaire liée à l'âge (DMLA) est la cause la plus fréquente de cécité non curable dans les pays industrialisés, avec une prévalence de $0,05 \%$ avant l'âge de 50 ans et de $11,8 \%$ après 80 ans. On estime qu'environ $65 \%$ des patients de 80 ans ou plus ont des signes précoces ou avancés de la maladie [1]. Les patients conservent cependant une autonomie ambulatoire puisque seule la macula est le siège de lésions. Sur le plan physiopathogénique, les signes précoces associent une altération des cellules de l'épithélium pigmenté de la rétine et des dépôts sous-épithéliaux appelés drüsens, composés entre autres de glycoprotéines, apolipoprotéine $\varepsilon$, vitronectine, et protéines de la cascade inflammatoire (composant amyloïde $P$, facteurs du complément, protéases). Une perte des photorécepteurs sus-jacents et de la choriocapillaire sous-jacente est aussi observée (Figure 1). L'évolution se fait vers une atrophie géographique, forme à progression lente et sans traitement à ce jour, ou plus rarement (environ $20 \%$ des cas) vers une néovascularisation (NV) sous-rétinienne, dite forme humide (ou exsudative) et d'aggravation rapide (Figure 2).

D'importantes avancées ont été faites au cours de ces deux dernières années tant dans la compréhension des mécanismes physiopathologiques que dans le traitement de la DMLA. C'est tout d'abord la mise en évidence du rôle de l'inflammation dans une pathologie considérée comme dégénérative et liée à l'âge. Un polymorphisme d'un nucléotide dans les gènes codant pour les facteurs $\mathrm{H}$ (CFH) (chromosome lq31), B (CFB) et C2 du complément est associé à 50 à $70 \%$ des cas de DMLA, quelle qu'en soit la forme. La régulation de l'activation de la voie alterne du complément serait modifiée par le produit de ces gènes [1-3]. Plus récemment, il a été montré qu'un polymorphisme d'un nucléotide dans la région promotrice du gène HTRAI (chromosome 10q26) conférerait un risque de $49,3 \%$ de DMLA. HTRAI code pour une sérine protéase de choc thermique, activée par le stress cellulaire et exprimée dans la rétine humaine et dans

les drüsens. Ce polymorphisme pourrait entraîner une augmentation locale de la protéase, favorisant les lésions tissulaires et la croissance des néo-vaisseaux $[4,5]$. De nombreux travaux restent à faire pour comprendre le sens réel de ces facteurs génétiques et leur implication dans l'apparition et l'évolution de la maladie.

Depuis les années 2000, la néovascularisation associée à la forme humide de DMLA est traitée par photothérapie dynamique (PDT). Un composé photo-

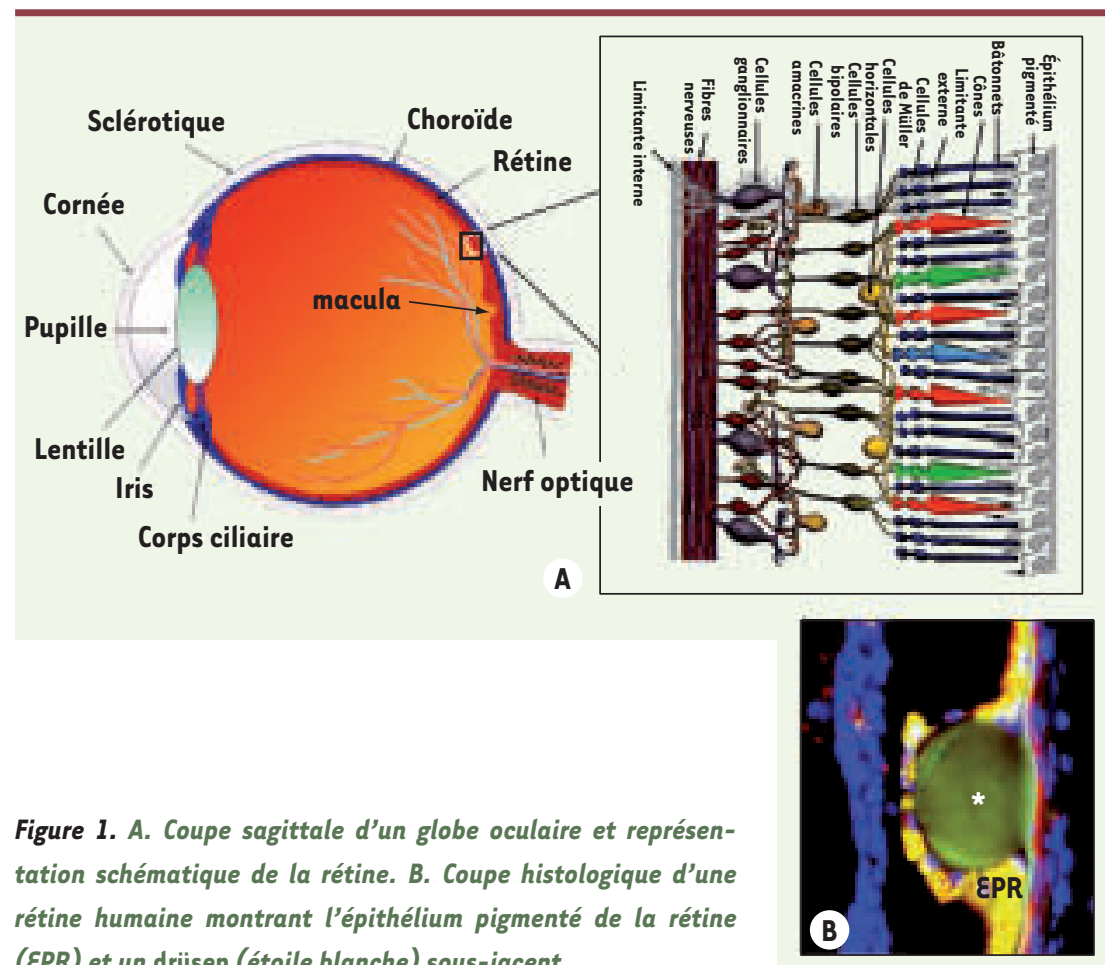


sensibilisant hydrophobe, formulé dans des liposomes (verteporphine, Visudyne ${ }^{\circledR}$, Novartis) est injecté par voie veineuse et activé spécifiquement dans la région maculaire par illumination avec un laser infrarouge. Les radicaux libres produits localement entraînent des lésions microvasculaires et une occlusion secondaire des néovaisseaux. Plusieurs formes cliniques de NV ont été décrites, celles où les néo-vaisseaux sont visibles à l'angiographie (forme dite à prédominance classique), et celles où les néo-vaisseaux sont occultes. La PDT est plus efficace dans certaines de ces formes. Dans les formes de $N V$ «à prédominance classique», la perte d'acuité visuelle (mesurée par la perte de reconnaissance des lettres) à deux ans d'intervalle est inférieure à 15 lettres dans $59 \%$ des cas traités par PDT, contre $31 \%$ dans le groupe non traité [6]. Ce résultat est confirmé par une étude de phase IV portant sur 4051 patients, qui rapporte cependant une perte de l'acuité visuelle dans 2,6\% des cas [7].

Plus récemment, deux molécules de nature différente, mais ayant toutes deux pour cible le VEGF (vascular endothelial growth factor), ont été mises sur le marché aux États-Unis pour traiter les NV. Le VEGF est en effet l'un des facteurs qui participent à la croissance des NV et favorisent leur perméabilité. L'inhibition du VEGF, soit par un oligoaptamère, c'est-à-dire un petit fragment de nucléotides qui se lie spécifiquement au VEGF (pegaptanib, Macugen ${ }^{\circledR}$, Pfizer), ou par un fragment Fab d'anticorps humanisé, dirigé contre le VEGF (Ranibizumab, Lucentis $^{\circledR}$, Novartis), a permis, pour la première fois, d'apporter non seulement une stabilisation de la vision mais également une amélioration (Figure 3). La principale différence entre ces deux molécules est un ciblage spécifique du VEGF $_{165}$ pour le Macugen ${ }^{\circledR}$, et un ciblage de toutes les isoformes du VEGF-A pour le Lucentis ${ }^{\circledR}$. Le premier produit mis sur le marché a été le Macugen et une étude en double-aveugle a montré que I'oligoaptamère injecté directement dans le globe oculaire (injection intravitréenne) toutes les 6 semaines permettait de stabiliser la vision (perte de moins de 15 lettres) à 54 semaines dans $70 \%$ des cas alors que, dans le groupe témoin, la stabilisation n'était obtenue que dans $55 \%$ des cas. Par ailleurs, les patients traités avaient une amélioration de la vision par rapport au groupe témoin tout au long du

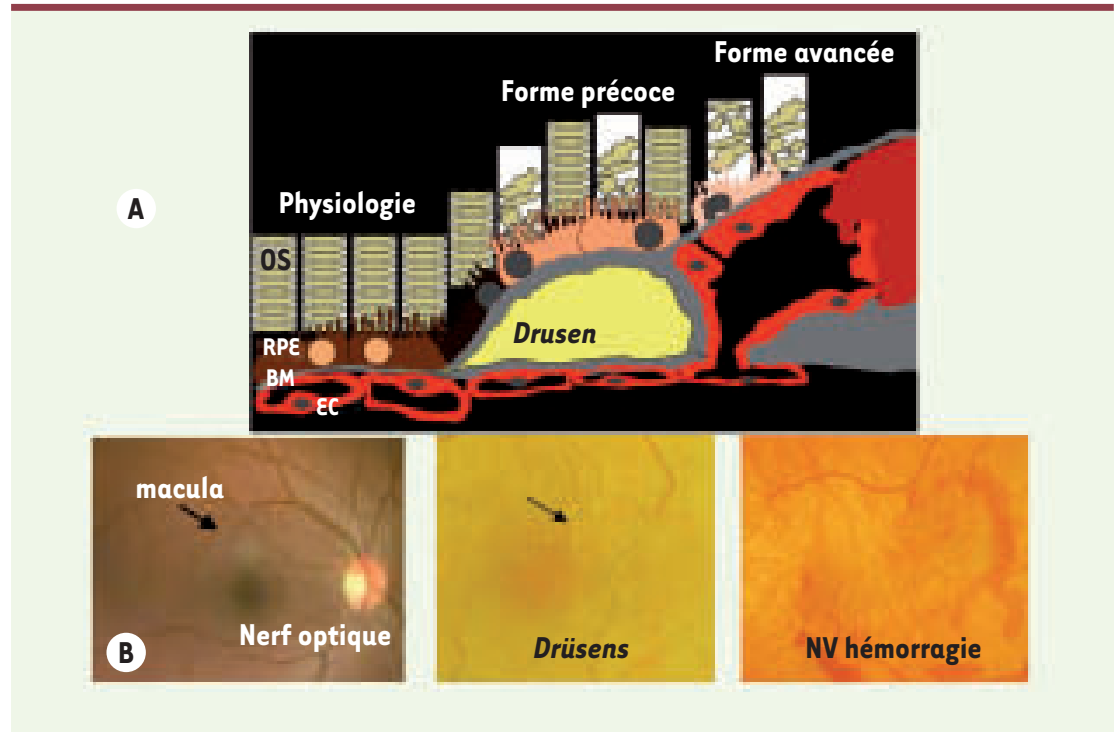

Figure 2. Phases évolutives de la DMLA ( $A$ ) et sa correspondance après examen du fond d'œil (B). $O S$ : segment externe des photorécepteurs; RPE : cellules pigmentées de la rétine; BM : membrane de Bruch. suivi [8]. En novembre 2006, les résultats de l'étude multicentrique randomisée évaluant l'efficacité du Lucentis ${ }^{\circledR}$ dans le traitement des NV de la DMLA est parue dans le New England Journal of Medicine. Parmi les patients ayant reçu toutes les 4 semaines une injection dans le globe oculaire de $0,3 \mathrm{mg}$ ou $0,5 \mathrm{mg}$ d'anticorps anti-VEGF, $94,5 \%$ et $94,6 \%$ d'entre eux respectivement avaient une perte d'acuité visuelle inférieure à 15 lettres, contre $62,2 \%$ dans le groupe témoin. Une augmentation de la vision de plus de 15 lettres a été observée au bout de deux ans chez $24,8 \%$ des patients traités avec $0,3 \mathrm{mg}$ et $33,8 \%$ des patients traités avec $0,5 \mathrm{mg}$ de Lucentis $^{\circledR}$, alors que seulement $5 \%$ des patients du groupe témoin avaient une amélioration spontanée de plus de 15 lettres [9]. Ces deux types de traitements comportent des risques d'infection oculaire sévère, liés aux injections intravitréennes, mais ils sont rares (1 à 1,7\% des patients), et des risques d'inflammation oculaire pour le Lucentis (5\%).

Dans l'étude MARINA, évaluant l'efficacité du Lucentis ${ }^{\circledR}$, des accidents vasculaires cérébraux ont été observés chez $2,5 \%$ des patients ayant reçu une injection de $0,5 \mathrm{mg}$ et chez $0,8 \%$ des patients du groupe témoin, et des hémorragies extra-oculaires ont été rapportées chez 9,2\% des patients ayant reçu une dose plus faible $(0,3 \mathrm{mg})$ contre $5,5 \%$ dans le groupe témoin. Ces effets connus des anti-VEGF administrés par voie systémique ont été observés avec la molécule qui bloque toutes les isoformes du VEGF-A. Ces traitements, dont le coût est élevé (entre 1000 et 1500 euros par injection) doivent être administrés toutes les 4 à 6 semaines pendant plusieurs années et pour une durée encore non déterminée. L'arrêt du traitement conduit à la récidive des signes exsudatifs.

Afin de réduire le coût des traitements, les ophtalmologistes ont tenté d'utiliser I'Avastin $^{\circledR}$ (bevacizumab, Genentech ${ }^{\circledR}$ ) pour l'injection intravitréenne. Cet anticorps anti-VEGF humanisé se présente sous la forme d'une immunoglobuline 
entière, et est utilisé en cancérologie. Ce produit est utilisé hors autorisation en milieu hospitalier à un coût très réduit ; les premiers résultats semblent montrer que l'Avastin ${ }^{\circledR}$ pourrait avoir des effets comparables à ceux du Lucentis ${ }^{\circledR}$, tout au moins à court terme [10].

Ces nouveaux travaux et traitements apportent de véritables espoirs pour les patients atteints de DMLA. Dans notre engouement et notre désir de procurer une amélioration visuelle à nos patients, restons cependant attentifs, en par- ticulier quant aux effets systémiques possibles d'une inhibition très prolongée du VEGF chez des patients âgés, et souvent porteurs de pathologies cardiovasculaires. Même injectés dans l'œil et à faibles doses, ces produits diffusent dans la circulation générale, induisant des risques cumulés quand les injections sont répétées.

L'avenir proche laisse entrevoir la possibilité de traitements combinés et l'utilisation séquentielle des différents produits disponibles: phase d'amorçage

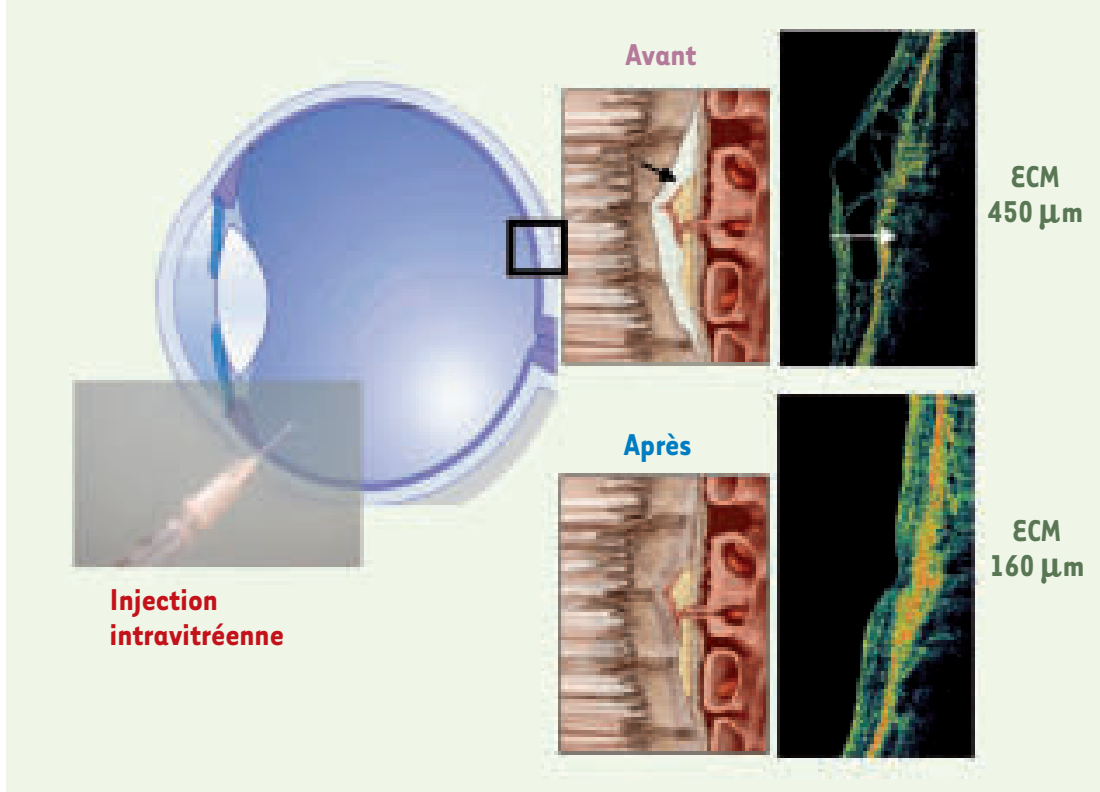

Figure 3. Injection intravitréenne et effet d'un traitement anti-angiogénique sur l'exsudat associé à la NV. Les représentations schématiques sont associées à des images en tomographie à cohérence optique obtenues chez un patient au cours du suivi. Ces images montrent une réduction de l'épaisseur maculaire centrale de $450 \mu \mathrm{m}$ à $160 \mu \mathrm{m}$ une semaine après injection d'un anti-VEGF.

avec les anti-VEGF les plus puissants (Lucentis $^{\circledR}$ ou $\operatorname{Avastin}^{\circledR}$ ), associés ou non à une PDT et relais avec des molécules plus sélectives comme le Macugen ${ }^{\circledR}$ pour la phase d'entretien. $\diamond$

Age-related macular degeneration:

therapeutic hopes

\section{RÉFÉRENCES}

1. De Jong PT. Age-related macular degeneration. $N$ Engl J Med 2006; 355 : 1474-85.

2. Haines JL, Hauser MA, Schmidt S, et al. Complement factor $\mathrm{H}$ variant increases the risk of age-related macular degeneration. Science $2005 ; 308$ : 418-21.

3. Klein RJ, Zeiss C, Chew $\varepsilon y$, et al. Complement factor $H$ polymorphisms in age-related macular degeneration. Science 2005 ; 308 : $385-9$.

4. Yang Z, Camp NJ, Sun H, et al. A variant of the HTRAl gene increases susceptibility to age-related macular degeneration. Science 2006 ; 314 : 992-3.

5. Dewan A, Liu M, Hartman S, et al. HTRAl promoter polymorphism in wet age-related macular degeneration. Science 2006 ; 314 : 989-92.

6. Arnold JJ, Blinder KJ, Bressler NM, et al. Treatment of age-related macular degeneration with photodynamic therapy. Verteporfin in photodynamic therapy study group. Acute severe visual acuity decrease after photodynamic therapy with verteporfin: case reports from randomized clinical trials-TAP and VIP report $n^{\circ} 3$. Am J Ophthalmol 2004 ; $137: 683-96$.

7. Meads C, Hyde C. Photodynamic therapy with verteporfin is effective, but how big is its effect? Results of a systematic review. BrJ Ophthalmol 2004 ; $88: 212-7$.

8. Kourlas H, Schiller DS. Pegaptanib sodium for the treatment of neovascular age-related macular degeneration: a review. Clin Ther 2006 ; 28 : 36-44.

9. Rosenfeld PJ, Brown DM, Heier JS, et al. for the MARINA study group. Ranibizumab for neovascular age-related macular degeneration. $N$ Engl J Med 2006 ; 335 : 1419-31.

10. Costa RA, Jorge R, Calucci D, et al. Intravitreal bevacizumab for choroidal neovascularization caused by AMD (IBeNA Study): results of a phase 1 doseescalation study. Invest Ophthalmol Vis Sci 2006 ; $47: 4569-78$

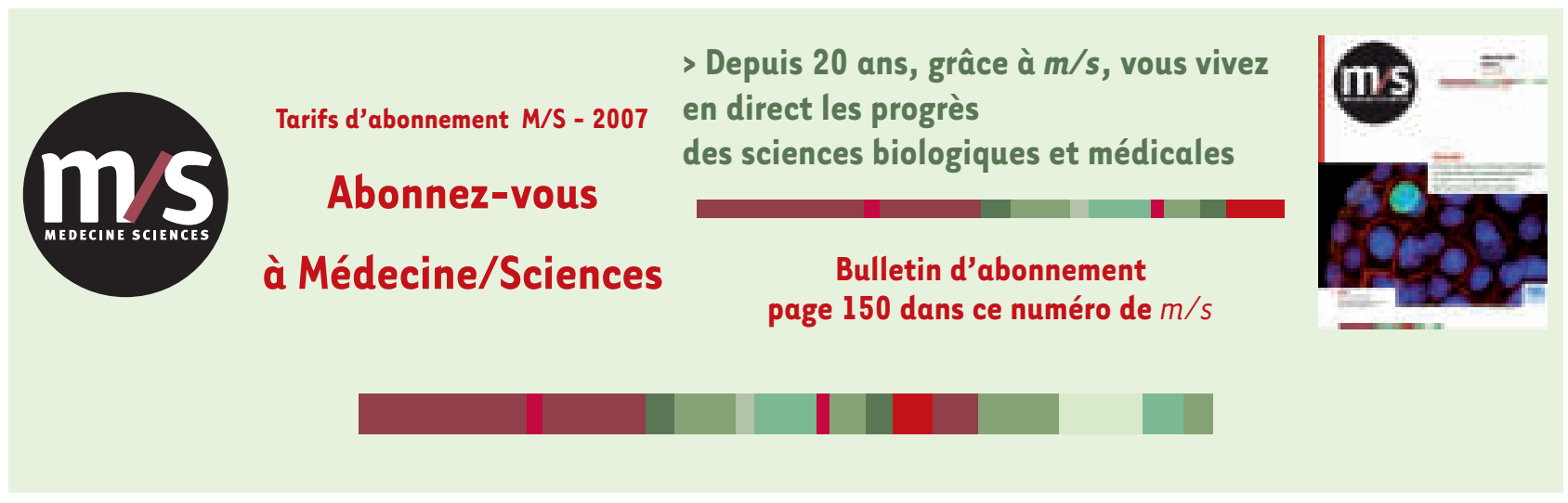

